\title{
Performance of IFN-Gamma release assays for detection of tuberculosis infection in juvenile I JIA children in biological therapy L Assante $^{1}, \mathrm{M}$ Bocchino $^{2}, \mathrm{M}$ Alessio*1, R Ambrosio ${ }^{1}$, A Sanduzzi $^{2}$ and A Guarino ${ }^{1}$
}

\begin{abstract}
Address: ${ }^{1}$ Department of Pediatrics Federico II University, Naples, Italy and ${ }^{2}$ Division of Respiratory Medicine, Federico II University, Naples, Italy
\end{abstract} * Corresponding author

from $15^{\text {th }}$ Paediatric Rheumatology European Society (PreS) Congress

London, UK. 14-17 September 2008

Published: 15 September 2008

Pediatric Rheumatology 2008, 6(Suppl I):P60 doi:10.1 I86/I546-0096-6-SI-P60

This abstract is available from: http://www.ped-rheum.com/content/6/SI/P60

(c) 2008 Assante et al; licensee BioMed Central Ltd.

Severe TB has recently been described in association with TNF- $\alpha$ blocker therapy. Furthermore conventional tuberculin skin test (TST) may give false-negative results in patients receiving biological therapy. New in vitro test, based on the detection of IFN- $\gamma$ release by T-cell stimulated with $M$. tuberculosis specific antigens (IGRA), are available for LTBI diagnosis, promising to be more sensitive and specific than TST. Nevertheless no sufficient evidence on IGRA performance are available in children. The performance of two commercially available IGRAs, ELISPOT-TB (TS-TB) and Quantiferon-TB gold in-Tube (QFTGIT), were evaluated in comparison with TST in children with rheumatic diseases. In children with positive result clinical and radiological evidence of TB was sought. Nineteen children with JIA were enrolled since November 2006 (median age 13.5 years; range 2-18). Seventeen children were on long term etanercept therapy plus methotrexate $(15 \mathrm{mg} / \mathrm{m} 2 /$ week $), 1$ on infliximab and 1 received adalimubab plus prednisone $(0,2 \mathrm{mg} / \mathrm{Kg} / \mathrm{die})$. Five children (26\%) showed positive TS-TB, QFT-GIT and TST were negative in all children. Both QFT-GIT and TS-TB showed a high percentage of indeterminate results $(21 \%$ and $16 \%$ respectively). None of the 5 patients positive at TS-TB developed active disease, as judged by clinical and radiological findings, at least in a median follow-up period of 16 months (range 9-17 months). Our data suggest that high rate of positive results by TS-TB is expression of non-specific immune reactivity in rheumatic children. 